\title{
A Multicenter Study of Invasive Fungal Infections in Patients with Childhood-onset Systemic Lupus Erythematosus
}

\author{
Marco F. Silva, Mariana P. Ferriani, Maria T. Terreri, Rosa M. Pereira, Claudia S. Magalhães, \\ Eloisa Bonfá, Lucia M. Campos, Eunice M. Okuda, Simone Appenzeller, Virgínia P. Ferriani, \\ Cássia M. Barbosa, Valéria C. Ramos, Simone Lotufo, and Clovis A. Silva
}

ABSTRACT. Objective. To study the prevalence, risk factors, and mortality of invasive fungal infections (IFI) in patients with childhood-onset systemic lupus erythematosus (cSLE).

Methods. A retrospective multicenter cohort study was performed in 852 patients with cSLE from 10 pediatric rheumatology services. An investigator meeting was held and all participants received database training. IFI were diagnosed according to the European Organization for Research and Treatment of Cancer/Invasive Fungal Infections Cooperative Group and the National Institute of Allergy and Infectious Diseases Mycoses Study Group Consensus Group criteria (proven, probable, and possible). Also evaluated were demographic, clinical, and laboratory data, and disease activity [SLE Disease Activity Index 2000 (SLEDAI-2K)], cumulative damage (Systemic Lupus International Collaborating Clinics/American College of Rheumatology Damage Index), treatment, and outcomes. Results. IFI were observed in 33/852 patients (3.9\%) with cSLE. Proven IFI was diagnosed in 22 patients with cSLE, probable IFI in 5, and possible IFI in 6. Types of IFI were candidiasis (20), aspergillosis (9), cryptococcosis (2), and 1 each disseminated histoplasmosis and paracoccidioidomycosis. The median of disease duration was lower (1.0 vs $4.7 \mathrm{yrs}, \mathrm{p}<0.0001)$ with a higher current SLEDAI-2K [19.5 (0-44) vs $2(0-45), \mathrm{p}<0.0001]$ and current prednisone (PRED) dose [50 (10-60) vs $10(2-90) \mathrm{mg} /$ day, $\mathrm{p}<0.0001$ ] in patients with IFI compared with those without IFI. The frequency of death was higher in the former group $(51 \%$ vs $6 \%, \mathrm{p}<0.0001)$. Logistic regression analysis revealed that SLEDAI-2K (OR 1.108, 95\% CI 1.057-1.163, p < 0.0001), current PRED dose (OR 1.046, 95\% CI $1.021-1.071, \mathrm{p}<0.0001)$, and disease duration (OR 0.984, 95\% CI 0.969-0.998, p = 0.030) were independent risk factors for IFI ( $\mathrm{R}^{2}$ Nagelkerke 0.425$)$.

Conclusion. To our knowledge, this is the first study to characterize IFI in patients with cSLE. We identified that disease activity and current glucocorticoid use were the main risk factors for these life-threatening infections, mainly in the first years of disease course, with a high rate of fatal outcome. (First Release November 15 2015; J Rheumatol 2015;42:2296-303; doi:10.3899/jrheum.150142)

Key Indexing Terms:

$\begin{array}{lrr}\text { INFECTION } & \text { INVASIVE FUNGAL INFECTION } & \text { INVASIVE FUNGAL DISEASE } \\ \text { CHILDHOOD-ONSET SYSTEMIC LUPUS ERYTHEMATOSUS } & \text { MULTICENTER COHORT }\end{array}$

From the Pediatric Rheumatology Unit, and Division of Rheumatology, Faculdade de Medicina da Universidade de São Paulo; Pediatric Rheumatology Unit, Universidade Federal de São Paulo; Faculdade de Medicina de Botucatu, São Paulo State University; Irmandade da Santa Casa de Misericórdia de São Paulo; State University of Campinas; Ribeirão Preto Medical School, University of São Paulo; Hospital Infantil Darcy Vargas; Pontifical Catholic University of Sorocaba; Hospital Municipal Infantil Menino Jesus, São Paulo, Brazil.

Supported by grants from Conselho Nacional de Desenvolvimento Científico e Tecnológico (CNPq 301805/2013-0 to RMRP, 305068/2014-8 to EB, and 302724/2011-7 to CAS), Federico Foundation (to RMRP, EB, and CAS), and by Núcleo de Apoio à Pesquisa "Saúde da Criança e do Adolescente" da USP (NAP-CriAd) to CAS.

M.F. Silva, MD, Pediatric Rheumatology Unit, Faculdade de Medicina da Universidade de São Paulo; M.P. Ferriani, MD, Pediatric Rheumatology Unit, Faculdade de Medicina da Universidade de São Paulo; M.T. Terreri, $M D, P h D$, Pediatric Rheumatology Unit, Universidade Federal de São

\author{
Paulo; R.M. Pereira, MD, PhD, Division of Rheumatology, Faculdade de \\ Medicina da Universidade de São Paulo; C.S. Magalhães, MD, PhD, \\ Faculdade de Medicina de Botucatu, São Paulo State University; \\ E. Bonfá, MD, PhD, Division of Rheumatology, Faculdade de Medicina da \\ Universidade de São Paulo; L.M. Campos, MD, PhD, Pediatric \\ Rheumatology Unit, Faculdade de Medicina da Universidade de São Paulo; \\ E.M. Okuda, MD, PhD, Irmandade da Santa Casa de Misericórdia de São \\ Paulo; S. Appenzeller, MD, PhD, State University of Campinas; \\ V.P. Ferriani, MD, PhD, Ribeirão Preto Medical School, University of São \\ Paulo; C.M. Barbosa, MD, PhD, Hospital Infantil Darcy Vargas; V.C. \\ Ramos, MD, Pontifical Catholic University of Sorocaba; S. Lotufo, MD, \\ Hospital Municipal Infantil Menino Jesus; C.A. Silva, MD, PhD, Pediatric \\ Rheumatology Unit, Faculdade de Medicina da Universidade de São Paulo. \\ Address correspondence to Prof. C.A. Silva, Av. Dr. Eneas Carvalho \\ Aguiar, 647 - Cerqueira César, São Paulo - SP, Brazil, 05403-000. \\ E-mail: clovisaasilva@gmail.com \\ Accepted for publication August 17, 2015.
}


Childhood-onset systemic lupus erythematosus (cSLE) is a chronic multisystem disease that is characterized by the production of autoreactive antibodies and immunologic system abnormalities ${ }^{1}$, which may predispose these patients to various infections.

Indeed, infections are an important cause of morbidity and mortality in patients with $\mathrm{cSLE}^{2}$. The most important risk factors associated with severe infection in the SLE population are related to the disease itself (disease duration, disease activity, lymphopenia, leukopenia, and hypocomplementemia) and treatment (glucocorticoid and immunosuppressive drugs) $2,3,4,5$.

The majority of infections are caused by viruses and bacteria $^{2,3,4,6}$, and less frequently by opportunistic agents, such as fungi ${ }^{7}$. Fungal infections are mainly caused by Candida spp., Pneumocystis jirovecii (formerly Pneumocystis carinii), and Cryptococcus neoformans in adult-onset SLE $(\text { aSLE) })^{8}$.

However, studies solely evaluating invasive fungal infections (IFI) in patients with cSLE, such as candidiasis, aspergillosis, cryptococcosis, and histoplasmosis, are limited to case reports and case series $7,9,10,11,12$ without any systematic evaluation of the associated risk factors and outcomes in the cSLE population.

Therefore, the objective of our study was to assess IFI in a large cSLE population, evaluating the prevalence, outcome, and risk factors.

\section{MATERIALS AND METHODS}

Study design and patients. This is a retrospective multicenter cohort study including 1017 patients with cSLE followed in 10 pediatric rheumatology tertiary referral services of São Paulo state, Brazil. All patients fulfilled the American College of Rheumatology (ACR) criteria ${ }^{13}$, with disease onset prior to the age of $18^{14}$ and current age until 25 years. One hundred and sixty-five patients were excluded because of incomplete medical charts ( $n=96$ ), undifferentiated connective tissue disorder with 3 or fewer of the ACR criteria $(n=43)$, isolated cutaneous lupus erythematosus $(n=11)$, neonatal lupus erythematosus $(n=8)$, drug-induced lupus $(n=5)$, and other autoimmune diseases $(n=2)$. None had hematological cancer, bone marrow transplantation, or human immunodeficiency virus (HIV) infection. The remaining 852 patients with cSLE composed the study group.

IFI was diagnosed according to the European Organization for Research and Treatment of Cancer/Invasive Fungal Infections Cooperative Group and the National Institute of Allergy and Infectious Diseases Mycoses Study Group Consensus Group ${ }^{15,16}$, and oral and vulvovaginal candidiasis were excluded. Local ethics committees of each center approved our study.

An investigator meeting was held for our study on September 29, 2012, in São Paulo city to determine the protocol, clinical variables, disease activity, and damage tools scoring, as well as outcome variables. Data collection training was conducted locally by investigators in each of the centers and a unique database was built up by MFS and MPF. Data discrepancy was sorted out by 1 or more rounds of queries for accuracy. Data were collected between November 2012 and October 2014. Patient medical charts were carefully reviewed according to an extensive standardized protocol for demographic data, clinical features, laboratory findings, treatment data, outcomes, and IFI characteristics.

IFI. Three levels of IFI probability were established in immunocompromised patients: proven, probable, and possible ${ }^{15,16}$. Proven IFI demands isolation of the fungus in a tissue or isolation in a sterile specimen (with a clinical or radiological abnormality consistent with the infection disease process). Probable IFI requires the presence of 1 host factor (for example, neutropenia and/or use of immunosuppressive agents) and/or 1 clinical criterion [clinical symptoms or laboratory abnormalities, such as prolonged fever, hepatosplenomegaly, adenomegaly, respiratory and sinus infection, meningoencephalitis, osteomyelitis; computed tomography showing focal lesions in central nervous system (CNS); or magnetic resonance image showing meningeal enhancement] in conjunction with mycological evidence through direct (culture, cytology, or microscopy) or indirect tests (antigen detection or cell wall component). Possible IFI was defined as the presence of at least 1 host factor in conjunction with suggestive clinical or laboratory abnormalities in the absence of mycological evidence ${ }^{15}$. P. jirovecii infection is not included in these definitions.

The following IFI were assessed: candidiasis, aspergillosis, histoplasmosis, cryptococcosis, paracoccidioidomycosis, coccidioidomycosis, and mucormycosis. IFI type, site of infection, fungus isolation, and antifungal therapy were also evaluated.

Demographic data, clinical evaluation, disease activity, disease damage, and therapy. Demographic data included sex, ethnicity, current age, age at cSLE onset, and disease duration. SLE clinical manifestations were defined as constitutional symptoms, involvement of the reticuloendothelial system, mucocutaneous lesions, musculoskeletal involvement, serositis, nephritis, and hematologic abnormalities. Neuropsychiatric lupus included 19 syndromes according to the ACR classification criteria ${ }^{17}$. Antiphospholipid syndrome was diagnosed according to the presence of arterial and/or venous thrombosis and antiphospholipid antibodies ${ }^{18}$.

High blood pressure was defined as systolic and/or diastolic blood pressures $\geq 95$ th percentile for sex, age, and height on $\geq 3$ occasions ${ }^{19}$. Acute kidney injury was determined by sudden increase in serum creatinine above $2 \mathrm{mg} / \mathrm{dl}^{20}$ or by the modified RIFLE criteria (Risk, Injury, Failure, Loss of kidney function, and End-stage kidney disease ${ }^{21}$. Chronic renal disease was defined as structural or functional abnormalities of the kidney for $\geq 3$ months [with or without decreased glomerular filtration rate (GFR)] or GFR $<60$ $\mathrm{ml} / \mathrm{min} / 1.73 \mathrm{~m}^{2}$ for $\geq 3$ months $^{22}$. Renal replacement therapy (hemodialysis, peritoneal dialysis, and hemofiltration and renal transplantation) was also assessed.

Laboratory assessment included erythrocyte sedimentation rate, C-reactive protein, complete blood cell count, serum urea and creatinine, urinalysis, and 24-h urine protein excretion. Complement levels (CH50, C3, and C4), anti-dsDNA, and anticardiolipin antibodies immunoglobulin (Ig) $\mathrm{G}$ and IgM were carried out at each center. The cutoff values were considered abnormal according to the kit manufacturer. Lupus anticoagulant was detected according to the guidelines of the International Society on Thrombosis and Hemostasis ${ }^{23}$.

SLE disease activity and cumulative damage were collected from the medical charts or retrospectively scored through the SLE Disease Activity Index 2000 (SLEDAI-2K) ${ }^{24}$ and the Systemic Lupus International Collaborating Clinics/ACR-Damage Index ${ }^{25}$, respectively.

Current treatment data [prednisone (PRED), methylprednisolone pulse therapy, chloroquine diphosphate, hydroxychloroquine sulfate, methotrexate, azathioprine, cyclosporine, mycophenolate mofetil, intravenous cyclophosphamide, rituximab, and plasmapheresis] were also recorded. Cumulative doses of PRED, intravenous methylprednisolone, glucocorticoid, and cyclophosphamide were calculated.

Patients were divided into 2 groups for the assessment of current SLE manifestations, laboratory abnormalities, and treatment: patients with IFI (evaluated at the infection diagnosis) and patients without IFI (evaluated at the last visit).

Statistical analysis. Results are presented as number (frequency) for categorical variables and median (range) or mean \pm SD for continuous variables. Categorical variables comparisons were assessed by Pearson chi-square or Fisher's exact test. Continuous variables from patients with cSLE with and without IFI were compared by Mann-Whitney U test or Student $\mathrm{t}$ test. The significance levels were set at $5 \%(\mathrm{p}<0.05)$.

Personal non-commercial use only. The Journal of Rheumatology Copyright $\odot$ 2015. All rights reserved 
Holm-Bonferroni correction for multiple comparisons was performed adjusting the significance level to $\mathrm{p}<0.0015$. Logistic regression analysis (backward stepwise) was performed to identify IFI risk factors.

\section{RESULTS}

IFI were recorded in 33/852 patients (3.9\%) with cSLE. Proven IFI was evidenced in 22 patients with cSLE, probable IFI in 5, and possible IFI in 6 . In 6 patients with cSLE, invasive fungal disease was identified at disease onset and the median SLEDAI-2K score was 28 (range 24-41). Types of IFI were candidiasis (20), aspergillosis (9), cryptococcosis ( 1 cryptococcal meningitis and 1 pulmonary cryptococcosis), and 1 each disseminated histoplasmosis and paracoccidioidomycosis. No patient had coccidioidomycosis and mucormycosis (Table 1). At least 1 site for IFI was evidenced in each patient: lungs in 13 , urinary tract in 8 , esophagitis in 8 , blood stream in $4, \mathrm{CNS}$ in 3 , and disseminated in 3 . Twenty-one were hospitalized in the pediatric intensive care units. The most frequent complications of IFI were sepsis in 18 and acute renal failure in 16 patients.

Of the 17 deaths $(51.5 \%), 15$ had sepsis and 14 had acute renal failure. Renal replacement therapy was required in 3 patients; all of them had invasive aspergillosis. The median survival time period from IFI diagnosis to death was 30 days (15-95). The most important types of IFI in patients with cSLE who died were aspergillosis (in 8) and candidiasis (in
7). Antibiotic use prior to fungal infection occurred in 21 patients $(63 \%)$, particularly in $8 / 9$ patients with aspergillosis $(88 \%)$ and 10/20 candidiasis (50\%), and in none of those with cryptococcosis. The median of PRED dose was significantly higher in patients with IFI who died compared with those who survived [60 (25-60) vs 40 (10-60) mg/day, $\mathrm{p}=0.014$ ].

Thirty-two patients received at least 1 antifungal therapy (26 fluconazole, 10 amphotericin B, 4 itraconazole, 3 voriconazole, 1 ketoconazole, and 1 micafungin). Only 1 patient did not receive antifungal therapy, because infection was diagnosed only at necropsy.

Shown in Table 2 are demographic data, clinical manifestations, disease activity/damage scores, and laboratory variables in 852 patients with cSLE according to IFI. After Holm-Bonferroni correction for multiple comparisons, disease duration (1.0 vs 4.7 yrs, p < 0.0001) and current age (14.5 vs 17.0 yrs, p < 0.0001 ) were both significantly lower in cSLE with IFI. The frequencies of these factors were significantly higher in patients with cSLE with IFI compared with those without IFI (all p < 0.0001): constitutional features or reticuloendothelial system involvement (76\% vs $8 \%$ ), mucocutaneous involvement (67\% vs $13 \%)$, musculoskeletal involvement (36\% vs 0\%), serositis (39\% vs 0\%), neuropsychiatric involvement (36\% vs $8 \%$ ), nephritis (66\% vs $19 \%)$, and arterial hypertension (58\% vs $12 \%)$. The median of SLEDAI-2K at cSLE onset [24 (2-45) vs 14 (0-58), p <

Table 1. IFI characteristics in 33/852 patients with cSLE.

\begin{tabular}{|c|c|c|c|}
\hline IFI Definitions & cSLE, $\mathrm{n}=33, \mathrm{n}(\%)$ & \multicolumn{2}{|c|}{ Mycological Criterion } \\
\hline Proven IFI & $22(67)$ & \multicolumn{2}{|c|}{ Isolation of the Fungus } \\
\hline \multirow[t]{6}{*}{ Candidiasis } & $13(40)$ & Urine culture, $\mathrm{n}=6$ & $\begin{array}{c}\text { Candida albicans, } \mathrm{n}=4 \\
\text { Candida } \text { spp., } \mathrm{n}=1\end{array}$ \\
\hline & & & Non-albicans yeast, $\mathrm{n}=1$ \\
\hline & & Blood culture, $\mathrm{n}=2$ & Candida albicans, $\mathrm{n}=1$ \\
\hline & & & Candida tropicalis, $\mathrm{n}=4$ \\
\hline & & Both urine and blood cultures, $\mathrm{n}=1$ & Candida albicans, $\mathrm{n}=1$ \\
\hline & & Both urine culture and bronchoalveolar lavage, $\mathrm{n}=1$ & Candida albicans, $\mathrm{n}=1$ \\
\hline \multirow[t]{4}{*}{ Aspergillosis } & $6(18)$ & Lung at necropsy, $\mathrm{n}=3$ & Aspergillus spp., $\mathrm{n}=3$ \\
\hline & & Lung and cerebellum at necropsy, $\mathrm{n}=1$ & Aspergillus spp., $\mathrm{n}=1$ \\
\hline & & Myocardium at necropsy, $\mathrm{n}=1$ & Aspergillus spp., $\mathrm{n}=1$ \\
\hline & & Multiple organs at necropsy, $\mathrm{n}=1$ & Aspergillus spp., $\mathrm{n}=1$ \\
\hline Candidiasis & $2(6)$ & Bronchoalveolar lavage, $\mathrm{n}=2$ & Candida albicans, $\mathrm{n}=2$ \\
\hline \multirow[t]{2}{*}{ Aspergillosis } & $2(6)$ & Indirect test, $\mathrm{n}=1$ & Increased serum galactomannan, $\mathrm{n}=1$ \\
\hline & & Bronchoalveolar lavage, $\mathrm{n}=1$ & Aspergillus fumigates, $\mathrm{n}=1$ \\
\hline Paracoccidioidomycosis & $1(3)$ & Indirect test, $\mathrm{n}=1$ & Counterimmunoelectrophoresis, $\mathrm{n}=1$ \\
\hline Possible IFI & $6(18)$ & \multicolumn{2}{|c|}{ Clinical or Laboratory Alterations in the Absence of Mycological Evidence } \\
\hline Candidiasis & $5(15)$ & \multicolumn{2}{|c|}{ Endoscopically confirmed Candida esophagitis, $\mathrm{n}=5$} \\
\hline Aspergillosis & $1(3)$ & \multicolumn{2}{|c|}{$\begin{array}{l}\text { Nodule with a halo of ground-glass attenuation in right lung image } \\
\text { (computerized tomography) with resolution after amphotericin B } \\
\text { and itraconazole, } \mathrm{n}=1\end{array}$} \\
\hline
\end{tabular}

IFI: invasive fungal infection; cSLE: childhood-onset systemic lupus erythematosus.

Personal non-commercial use only. The Journal of Rheumatology Copyright $\odot$ (2015. All rights reserved 
Table 2. Demographic data, clinical manifestations, disease activity/damage scores, and laboratory tests in 852 patients with cSLE grouped according to IFI. Values are $\mathrm{n}(\%)$ or median (range) unless otherwise specified.

\begin{tabular}{|c|c|c|c|}
\hline Variables & With IFI, $\mathrm{n}=33$ & Without IFI, $\mathrm{n}=819$ & $\mathrm{p}$ \\
\hline \multicolumn{4}{|l|}{ Demographic data } \\
\hline Female & $29(88)$ & $703(86)$ & 1.000 \\
\hline Age at cSLE onset, yrs, $\mathrm{n}=846$ & $12.4(5.1-17.2)$ & $11.8(0.2-17.8)$ & 0.159 \\
\hline Disease duration, yrs, $\mathrm{n}=846$ & $1.0(0-7.3)$ & $4.7(0-23.4)$ & $<0.0001^{*}$ \\
\hline Current age, yrs, $\mathrm{n}=849$ & $14.5(6-20)$ & $17.0(2-25.9)$ & $<0.0001^{*}$ \\
\hline Fever, $\mathrm{n}=849$ & $24 / 33(73)$ & $35 / 816(4)$ & $<0.0001^{*}$ \\
\hline Weight loss $>2 \mathrm{~kg}, \mathrm{n}=824$ & $8 / 33(24)$ & $25 / 791(3)$ & $<0.0001^{*}$ \\
\hline Lymphadenopathy, $\mathrm{n}=848$ & $6 / 32(19)$ & $8 / 816(1)$ & $<0.0001^{*}$ \\
\hline Hepatomegaly, $\mathrm{n}=850$ & $9 / 33(27)$ & $15 / 817(2)$ & $<0.0001^{*}$ \\
\hline Splenomegaly, $\mathrm{n}=850$ & $3 / 33(9)$ & $5 / 817(1)$ & 0.003 \\
\hline Mucocutaneous involvement, $\mathrm{n}=850$ & $22 / 33(67)$ & $108 / 817(13)$ & $<0.0001^{*}$ \\
\hline Current autoimmune thrombosis, APS, $\mathrm{n}=835$ & $3 / 33(9)$ & $10 / 802(1)$ & 0.012 \\
\hline \multicolumn{4}{|l|}{ Other } \\
\hline Arterial hypertension, $\mathrm{n}=843$ & $19 / 33(58)$ & $100 / 810(12)$ & $<0.0001 *$ \\
\hline Acute renal failure, $\mathrm{n}=841$ & $16 / 33(48)$ & $21 / 808(3)$ & $<0.0001^{*}$ \\
\hline Chronic renal failure, $\mathrm{n}=842$ & $3 / 33(9)$ & $24 / 809(3)$ & 0.084 \\
\hline Renal replacement therapy, $n=842$ & $3 / 33(9)$ & $25 / 809(3)$ & 0.092 \\
\hline Death, $\mathrm{n}=845$ & $17 / 33(51)$ & $50 / 812(6)$ & $<0.0001^{*}$ \\
\hline \multicolumn{4}{|l|}{ Disease activity/damage } \\
\hline SLEDAI-2K at cSLE onset, $\mathrm{n}=780 / 852$ & $24(2-45)$ & $14(0-58)$ & $<0.0001^{*}$ \\
\hline Current SLEDAI-2K, $\mathrm{n}=754 / 852$ & $19.5(0-44)$ & $2(0-45)$ & $<0.0001 *$ \\
\hline Current SLICC, $\mathrm{n}=822 / 852$ & $0(0-9)$ & $0(0-7)$ & 0.037 \\
\hline \multicolumn{4}{|l|}{ Current laboratory tests } \\
\hline Lupus anticoagulant, $\mathrm{n}=311$ & $3 / 14(21)$ & $43 / 297(14)$ & 0.444 \\
\hline $\mathrm{aCL} \operatorname{IgM}, \mathrm{n}=348$ & $3 / 15(20)$ & $51 / 333(15)$ & 0.712 \\
\hline aCL IgG, $n=349$ & $2 / 15(13)$ & $64 / 334(19)$ & 0.746 \\
\hline
\end{tabular}

* P value according to Bonferroni correction for multiple comparisons ( $\mathrm{p}<0.0015)$. cSLE: childhood-onset systemic lupus erythematosus; IFI: invasive fungal infection; APS: antiphospholipid syndrome; SLEDAI-2K: Systemic Lupus Erythematosus Disease Activity Index 2000; SLICC: Systemic Lupus International Collaborating Clinics; ESR: erythrocyte sedimentation rate; CRP: C-reactive protein; C3: complement factor 3; C4: complement factor 4; aCL: anticardiolipin; IgM: immunoglobulin M; IgG: immunoglobulin G.

$0.0001]$ and current SLEDAI-2K [19.5 (0-44) vs $2(0-45)$, $\mathrm{p}<0.0001]$ were significantly higher in patients with IFI. Renal failure $(48 \%$ vs $3 \%, \mathrm{p}<0.0001)$ and death $(51 \%$ vs $6 \%, \mathrm{p}<0.0001)$ were more frequently observed in patients with IFI.

Therapy of 852 patients with cSLE according to the IFI presence is illustrated in Table 3. After Holm-Bonferroni correction for multiple comparisons, frequencies of the following treatments were significantly higher in patients with IFI compared to those without: methylprednisolone pulse therapy (61\% vs $7 \%, \mathrm{p}<0.0001)$, intravenous cyclophosphamide ( $27 \%$ vs $5 \%, \mathrm{p}<0.0001$ ), and plasmapheresis $(12 \%$ vs $0 \%, \mathrm{p}<0.0001)$.

The median current PRED dose was also significantly higher in patients with cSLE with IFI in comparison to those without IFI [50 (10-60) vs 10 (2-90) mg/day, p < 0.0001]. These treatment doses were similar in patients with cSLE with IFI in comparison with those without IFI: PRED cumulative dose [15.77 (0.24-35.6) vs $19.08(0.12-105.51)$ $\mathrm{g}, \mathrm{p}=0.089]$, intravenous methylprednisolone cumulative dose [6.3 (2.0-35.3) vs $8.9(0.24-138.5) \mathrm{g}, \mathrm{p}=0.354$ ], and total glucocorticoid dose $\left[\begin{array}{lll}20.9 & (3.6-71.7) & \text { vs } 26.07\end{array}\right.$ 
Table 3. Current therapy of 852 patients with cSLE according to IFI. Values are n (\%) or median (range) unless otherwise specified.

\begin{tabular}{|c|c|c|c|}
\hline Variables & With IFI, $\mathrm{n}=33$ & Without IFI, $\mathrm{n}=819$ & $\mathrm{p}^{*}$ \\
\hline Nonsteroidal antiinflammatory, $\mathrm{n}=843$ & $3 / 33(9)$ & $43 / 810(5)$ & 0.418 \\
\hline \multicolumn{4}{|l|}{ Glucocorticoid } \\
\hline Current dose, $\mathrm{mg} /$ day, $\mathrm{n}=669$ & $50(10-60)$ & $10(2-90)$ & $<0.0001 *$ \\
\hline $\mathrm{mg} / \mathrm{kg} / \mathrm{day}, \mathrm{n}=648$ & $1.0(0.1-2.0)$ & $0.2(0.02-4.7)$ & $<0.0001^{*}$ \\
\hline Cumulative dose, $\mathrm{g}, \mathrm{n}=710$ & $15.77(0.24-35.6)$ & $19.08(0.12-105.51)$ & 0.089 \\
\hline Total glucocorticoid dose, $\mathrm{g}, \mathrm{n}=688$ & $20.9(3.6-71.7)$ & $26.07(0.42-205.54)$ & 0.285 \\
\hline Antimalarial drugs, $\mathrm{n}=841$ & $21 / 33(64)$ & $548 / 808(68)$ & 0.614 \\
\hline Chloroquine diphosphate, $\mathrm{n}=816$ & $8 / 33(24)$ & $123 / 783(16)$ & 0.191 \\
\hline Hydroxychloroquine sulfate, $\mathrm{n}=818$ & $13 / 33(39)$ & $440 / 785(56)$ & 0.059 \\
\hline Immunosuppressive agents, $\mathrm{n}=845$ & $23 / 33(70)$ & $499 / 812(61)$ & 0.339 \\
\hline Azathioprine, $\mathrm{n}=842$ & $14 / 33(42)$ & $284 / 809(35)$ & 0.389 \\
\hline Cumulative dose, $\mathrm{g}, \mathrm{n}=306$ & $2.8(0.6-12.8)$ & $7.0(0.26-93)$ & 0.002 \\
\hline \multicolumn{4}{|l|}{ Others } \\
\hline Rituximab, $\mathrm{n}=843$ & $1 / 33(3)$ & $1 / 810(0)$ & 0.077 \\
\hline Plasmapheresis, $\mathrm{n}=845$ & 4/33 (12) & $3 / 812(0)$ & $<0.0001^{*}$ \\
\hline
\end{tabular}

* P value according to Bonferroni correction for multiple comparisons $(\mathrm{p}<0.0015)$. cSLE: childhood-onset systemic lupus erythematosus; IFI: invasive fungal infection.

(0.42-205.54) g, $\mathrm{p}=0.285]$. After Holm-Bonferroni correction for multiple comparisons, the median of cyclophosphamide cumulative dose did not reach statistical significance in either group [2.8 $(0.6-12.8)$ vs $7.0(0.26-93) \mathrm{g}, \mathrm{p}=$ $0.002]$.

Logistic regression analysis revealed that these were independent risk factors for IFI: SLEDAI-2K (OR 1.108, 95\% CI 1.057-1.163, $\mathrm{p}<0.0001)$, current PRED dose in $\mathrm{mg} /$ day (OR $1.046,95 \%$ CI $1.021-1.071, \mathrm{p}<0.0001)$, and disease duration (OR 0.984, 95\% CI 0.969-0.998, $\mathrm{p}=0.030$ ). The $\mathrm{R}^{2}$ of Nagelkerke was 0.425 (Table 4).

The logistic regression analysis excluding cases that used cyclosporine showed that these were also independent risk factors for IFI: SLEDAI-2K (OR 1.126, 95\% CI $1.071-1.183, \mathrm{p}<0.0001$ ), current PRED dose in $\mathrm{mg} /$ day (OR $1.038,95 \%$ CI $1.014-1.064, \mathrm{p}=0.002)$, and disease duration (OR $0.983,95 \%$ CI $0.967-0.999, p=0.036$ ). The $\mathrm{R}^{2}$ of Nagelkerke was 0.416 .

Table 4. Stepwise logistic regression analysis to evaluate risk factors for IFI in 852 patients with cSLE.

\begin{tabular}{lcccc}
\hline Independent Variable & OR & $95 \% \mathrm{CI}$ & $\mathrm{R}^{2}$ & $\mathrm{p}$ \\
\hline Current SLEDAI-2K & 1.108 & $1.057-1.163$ & 0.425 & $<0.0001^{*}$ \\
PRED current dose, mg/day & 1.046 & $1.021-1.071$ & - & $<0.0001^{*}$ \\
Disease duration & 0.984 & $0.969-0.998$ & - & $0.030^{*}$
\end{tabular}

\footnotetext{
$* \mathrm{P}$ value $<0.05$. IFI: invasive fungal infection; cSLE: childhood-onset systemic lupus erythematosus; SLEDAI-2K: SLE Disease Activity Index 2000; PRED: prednisone.
}

The logistic regression analysis excluding cases that used cyclophosphamide revealed that these were independent risk factors for IFI: SLEDAI-2K (OR 1.112,95\% CI 1.056-1.171, $\mathrm{p}<0.0001$ ), current PRED dose in mg/day (OR 1.035, 95\% CI 1.009-1.060, $\mathrm{p}=0.007$ ), and disease duration (OR 0.981, $95 \%$ CI $0.965-0.996, \mathrm{p}=0.016)$. The $\mathrm{R}^{2}$ of Nagelkerke was 0.343 .

Further analysis of a subgroup of 33 patients with cSLE with IFI and 66 patients without IFI (randomly selected out of 819 cSLE) who presented similar disease duration [1 $(0-7.33)$ vs $1(0-7.33)$ yrs, $p=0.772]$ revealed in the former group a higher median of SLEDAI-2K [19.5 (0-44) vs 4 $(0-45), \mathrm{p}<0.0001]$ and current PRED dose [50 (10-60) vs $20(5-80) \mathrm{mg} / \mathrm{day}, \mathrm{p}<0.0001]$. The frequency of premature death was significantly higher in patients with cSLE with IFI compared with those without this infection $(51.5 \%$ vs $20 \%$, $\mathrm{p}=0.001)$.

\section{DISCUSSION}

Our study provided novel data demonstrating that invasive fungal diseases in patients with cSLE are life-threatening infections occurring predominantly in the first years of disease presentation.

The advantage of our present study was a large cohort, recorded consistently in 10 selected centers in São Paulo state, Brazil. To minimize bias, standard procedures were performed for data collection. The definition of IFI was based on expert opinion and international consensus ${ }^{15,16}$. These criteria have been used in clinical trials and epidemiological 
studies $^{26}$. Superficial candidiasis (such as oral and vulvovaginal $)^{15}$ were observed, but they were not included in the analysis of IFI because they are not invasive and are generally promptly treated.

The main limitations were the retrospective study design and missing data. A precise evaluation of Ig levels was not recorded, precluding a definitive interpretation regarding primary immunodeficiency associated with $\mathrm{cSLE}^{1,27}$. In addition, there were collinearity of the variables to differentiate between IFI and disease variables, in spite of using Holm-Bonferroni correction for multiple comparisons and logistic regression.

Major infections are also an important cause of morbidity and mortality in patients with cSLE, and they occur with high frequency, from $37 \%$ to $57 \%$ 2,4,5. Invasive fungal diseases occur more often in immunosuppressed patients with hematological cancer, bone marrow transplantation ${ }^{28}$, and HIV infection $^{29}$. The IFI frequency of $3.9 \%$ observed herein in patients with cSLE is similar to that reported for aSLE $(0.64-4.8 \%)^{29,30,31,32,33,34}$.

Of note, the majority of IFI observed in our present study occurred in the first years of disease. A systematic review of invasive fungal disease using the same criteria for categorizing these infections ${ }^{15}$ in patients with aSLE showed that IFI was diagnosed within a median of 2 years $^{29}$. About one-fifth of our patients with cSLE presented IFI at disease onset, differently from $9 \%$ of new-onset aSLE diagnosed concurrently with these infections ${ }^{29}$. Of note, all of them had severe disease activity (SLEDAI-2K > 20), reinforcing previous observations that infection can exacerbate or mimic disease activity, or induce flare ${ }^{4,9}$. Disease activity was also frequently reported at onset of IFI in patients with aSLE, similar to our population $26,30,31,32$.

Systemic Candida infections were observed in $2.35 \%$ of our patients with cSLE, a frequency comparable with that reported in the general population of 4 tertiary care hospitals in São Paulo, Brazil $(4 \%)^{35}$.

Patients with cSLE usually require higher steroid doses than patients with aSLE ${ }^{36}$. High current glucocorticoid dose was identified as one of the most important risk factors for IFI in cSLE without any association with cumulative glucocorticoid dose, and independent of cyclophosphamide and cyclosporine use. In spite of that, concomitant use of immunosuppressive agents in these patients was reported to be associated with cellular immunity impairment with consequent lymphopenia and risk of severe infection ${ }^{26}$.

High mortality rate was most likely related to sepsis and acute renal failure, an almost universal complication in the patients evaluated here ${ }^{30}$. Lower respiratory tract was the main site of IFI in children, whereas in adults there is a predominance of CNS involvement ${ }^{30}$. cSLE differed from aSLE regarding clinical syndromes of systemic mycosis. Candidiasis and aspergillosis were the majority of pediatric cases, in contrasting to adults, in whom cryptococcosis seems to be the main cause of $\mathrm{IFI}^{30,34,37}$. Moreover, the disease activity itself could present lymphopenia that may be aggravated by cytotoxic therapy.

Antibiotic use prior to IFI occurred in two-thirds of our patients, including almost the totality of patients with aspergillosis and half of patients with candidiasis, similar to the frequency reported in patients with aSLE ${ }^{38}$. Of note, cryptococcosis was not associated with this treatment. This finding may be related to the fact that aspergillosis and candidiasis are infections potentially acquired during hospitalization and therefore prior use of antibiotics is more likely than in cryptococcosis, a disease acquired mainly by inhalation of aerosolized particles associated with bird droppings, particularly pigeons, and bats ${ }^{10}$.

Antifungal therapy was promptly initiated in all patients but 1, particularly fluconazole for candidiasis and amphotericin $\mathrm{B}$ or voriconazole for other $\mathrm{IFI}^{30}$. In spite of that, half of our patients died, suggesting that new guidelines for prophylactic antifungal agents should be established in the cSLE population, as reported in pediatric cancer ${ }^{39,40}$.

To our knowledge, ours was the first study that characterized IFI in patients with cSLE. We identified that disease activity and glucocorticoid were the main risk factors for these life-threatening infections in early disease course with a high rate of fatal outcome.

\section{ACKNOWLEDGMENT}

Our gratitude to Ulysses Doria-Filho for the statistical analysis. The authors thank the following Pediatric Rheumatology Divisions and colleagues for including their patients: Pediatric Rheumatology Unit, Faculdade de Medicina da Universidade de São Paulo (FMUSP; Adriana Maluf Elisa Sallum, Cristina Miuki Abe Jacob, Gabriela Blay, Gabriela Nunes Leal, Gabriella Erlacher Lube de Almeida, Heloisa Helena de Souza Marques, João Domingos Montoni da Silva, Joaquim Carlos Rodrigues, Juliana Caíres de Oliveira Achili Ferreira, Kátia Kozu, Laila Pinto Coelho, Luciana dos Santos Henriques, Magda Carneiro-Sampaio, Maria Helena Vaisbich, Nadia Emi Aikawa, Roberta Cunha Gomes, Victor Leonardo Saraiva Marques, Werther Brunow de Carvalho); Pediatric Rheumatology Unit, Universidade de São Paulo (Ana Paula Sakamoto, Anandreia Simões Lopes, Claudio Arnaldo Len, Daniela Petry Piotto, Giampaolo Faquin, Gleice Clemente, Maria Odete Esteves Hilário, Melissa Fraga, Octavio Augusto Bedin Peracchi, Vanessa Bugni); Division of Rheumatology, FMUSP (Juliane A Paupitz, Glauce Leão Lima); Universidade Estadual de São Paulo (Priscila R. Aoki, Juliana de Oliveira Sato, Silvana Paula Cardin, Taciana Albuquerque Pedrosa Fernandes), Irmandade da Santa Casa de Misericórdia de São Paulo (Andressa Guariento, Eunice Okuda, Maria Carolina dos Santos, Natali Weniger Spelling Gormenzano, Silvana Brasília Sacchetti), State University of Campinas (Marisa Centeville, Renata Barbosa, Roberto Marini), Ribeirão Preto Medical School - University of São Paulo (Francisco Hugo Gomes, Gecilmara Salviatto Pileggi, Luciana Martins de Carvalho, Paola Pontes Pinheiro), Hospital Infantil Darcy Vargas (Jonatas Libório, Luciana Tudech Pedro Paulo), and Hospital Municipal Infantil Menino Jesus (Tânia Caroline Monteiro de Castro).

\section{REFERENCES}

1. Jesus AA, Liphaus BL, Silva CA, Bando SY, Andrade LE, Coutinho A, et al. Complement and antibody primary immunodeficiency in juvenile systemic lupus erythematosus patients. Lupus 2011;20:1275-84

Personal non-commercial use only. The Journal of Rheumatology Copyright @ $\odot 2015$. All rights reserved 
2. Lee PP, Lee TL, Ho MH, Wong WH, Lau YL. Recurrent major infections in juvenile-onset systemic lupus erythematosus - a close link with long-term disease damage. Rheumatology 2007; 46:1290-6.

3. Al-Mayouf SM, Al-Jumaah S, Bahabri S, Al-Eid W. Infections associated with juvenile systemic lupus erythematosus. Clin Exp Rheumatol 2001;19:748-50.

4. Faco MM, Leone C, Campos LM, Febrônio MV, Marques HH, Silva CA. Risk factors associated with the death of patients hospitalized for juvenile systemic lupus erythematosus. Braz J Med Biol Res 2007;40:993-1002.

5. Costa-Reis P, Nativ S, Isgro J, Rodrigues T, Yildirim-Toruner C, Starr A, et al. Major infections in a cohort of 120 patients with juvenile-onset systemic lupus erythematosus. Clin Immunol 2013;149:442-9.

6. Lube G, Aikawa NE, Tacla M, Leal MM, Lourenço B, Silva LE, et al. Condyloma acuminatum by human papilloma virus infection in childhood-systemic lupus erythematosus patients. Acta Reumatol Port 2014;39:182-7.

7. Canova EG, Rosa DC, Vallada MG, Silva CA. Invasive aspergillosis in juvenile systemic lupus erythematosus. A clinico-pathologic case. Clin Exp Rheumatol 2002;20:736.

8. Danza A, Ruiz-Irastorza G. Infection risk in systemic lupus erythematosus patients: susceptibility factors and preventive strategies. Lupus 2013;22:1286-94.

9. Silva MF, Ribeiro AS, Fiorot FJ, Aikawa NE, Lotito AP, Campos LM, et al. Invasive aspergillosis: a severe infection in juvenile systemic lupus erythematosus patients. Lupus 2012;21:1011-6.

10. Cavalcante EG, Montoni JD, Oliveira GT, Campos LM, Paz JA, Silva CA. Fatal cryptococcal meningitis in a juvenile lupus erythematosus patient. Rev Bras Reumatol 2014;54:155-8.

11. Marques VL, Gomes RC, Viola GR, Maia MM, Durigon GS, Aikawa NE, et al. Pulmonary cryptococcosis in childhood systemic lupus erythematosus and Sjögren syndrome overlap: a rare opportunistic infection. Lupus 2013;22:1409-12.

12. França CM, Cavalcante EG, Ribeiro AS, Oliveira GT, Litvinov N, Silva CA. Disseminated histoplasmosis in a juvenile lupus erythematosus patient. Acta Reumatol Port 2012;37:276-9.

13. Hochberg MC. Updating the American College of Rheumatology revised criteria for the classification of systemic lupus erythematosus. Arthritis Rheum 1997;40:1725.

14. Silva CA, Avcin T, Brunner HI. Taxonomy for systemic lupus erythematosus with onset before adulthood. Arthritis Care Res 2012;64:1787-93.

15. De Pauw B, Walsh TJ, Donnelly JP, Stevens DA, Edwards JE, Calandra T; European Organization for Research and Treatment of Cancer/Invasive Fungal Infections Cooperative Group; National Institute of Allergy and Infectious Diseases Mycoses Study Group (EORTC/MSG) Consensus Group. Revised definitions of invasive fungal disease from the European Organization for Research and Treatment of Cancer/Invasive Fungal Infections Cooperative Group and the National Institute of Allergy and Infectious Diseases Mycoses Study Group (EORTC/MSG) Consensus Group. Clin Infect Dis 2008;46:1813-21.

16. Hoenigl M, Strenger V, Buzina W, Valentin T, Koidl C, Wölfler A. European Organization for the Research and Treatment of Cancer/Mycoses Study Group (EORTC/MSG) host factors and invasive fungal infections in patients with haematological malignancies. J Antimicrob Chemother 2012;67:2029-33.

17. The American College of Rheumatology nomenclature and case definitions for neuropsychiatric lupus syndromes. Arthritis Rheum 1999;42:599-608.

18. Avcin T, Cimaz R, Rozman B; Ped-APS Registry Collaborative Group. The Ped-APS Registry: the antiphospholipid syndrome in childhood. Lupus 2009;18:894-9.
19. National High Blood Pressure Education Program Working Group on High Blood Pressure in Children and Adolescents. The fourth report on the diagnosis, evaluation, and treatment of high blood pressure in children and adolescents. Pediatrics 2004;114:555-76.

20. Chan JC, Williams DM, Roth KS. Kidney failure in infants and children. Pediatr Rev 2002;23:47-60.

21. Akcan-Arikan A, Zappitelli M, Loftis LL, Washburn KK, Jefferson LS, Goldstein SL. Modified RIFLE criteria in critically ill children with acute kidney injury. Kidney Int 2007;71:1028-35.

22. National Kidney Foundation. K/DOQI clinical practice guidelines for chronic kidney disease: evaluation, classification, and stratification. Am J Kidney Dis 2002;39 Suppl 1:S1-266.

23. Brandt JT, Triplett DA, Alving B, Scharrer I. Criteria for the diagnosis of lupus anticoagulants: an update. On behalf of the Subcommittee on Lupus Anticoagulant/Antiphospholipid Antibody of the Scientific and Standardisation Committee of the ISTH. Thromb Haemost 1995;74:1185-90.

24. Gladman DD, Ibañez D, Urowitz MB. Systemic lupus erythematosus disease activity index 2000. J Rheumatol 2002;29:288-91.

25. Gladman D, Ginzler E, Goldsmith C, Fortin P, Liang M, Urowitz M, et al. The development and initial validation of the Systemic Lupus International Collaborating Clinics/American College of Rheumatology damage index for systemic lupus erythematosus. Arthritis Rheum 1996;39:363-9.

26. Barber CE, Barnabe C. Another consequence of severe lupus: invasive fungal disease. J Rheumatol 2012;39:1772-4.

27. Jesus AA, Jacob CM, Silva CA, Dorna M, Pastorino AC, Carneiro-Sampaio M. Common variable immunodeficiency associated with hepatosplenic T-cell lymphoma mimicking juvenile systemic lupus erythematosus. Clin Dev Immunol 2011;2011:428703.

28. Ozsevik SN, Sensoy G, Karli A, Albayrak C, Dagdemir A, Belet N. Invasive fungal infections in children with hematologic and malignant diseases. J Pediatr Hematol Oncol 2015;37:e69-72.

29. Wang LR, Barber CE, Johnson AS, Barnabe C. Invasive fungal disease in systemic lupus erythematosus: a systematic review of disease characteristics, risk factors, and prognosis. Semin Arthritis Rheum 2014;44:325-30.

30. Chen HS, Tsai WP, Leu HS, Ho HH, Liou LB. Invasive fungal infection in systemic lupus erythematosus: an analysis of 15 cases and a literature review. Rheumatology 2007;46:539-44.

31. Kim HJ, Park YJ, Kim WU, Park SH, Cho CS. Invasive fungal infections in patients with systemic lupus erythematosus: experience from affiliated hospitals of Catholic University of Korea. Lupus 2009;18:661-6

32. Fan YC, Li WG, Zheng MH, Gao W, Zhang YY, Song LJ. Invasive fungal infection in patients with systemic lupus erythematosus: experience from a single institute of Northern China. Gene 2012;506:184-7.

33. Martínez-Martínez MU, Herrera-Van Oostdam D, Román-Acosta S, Magaña-Aquino M, Baranda-Cándido L, Abud-Mendoza C. Invasive fungal infections in patients with systemic lupus erythematosus. J Rheumatol 2012;39:1814-8.

34. Vinicki JP, Catalan Pellet S, Pappalardo C, Cruzat VC, Spinetto MA, Dubinsky D. Invasive fungal infections in Argentine patients with systemic lupus erythematosus. Lupus 2013;22:892-8.

35. Colombo AL, Guimarães T, Silva LR, de Almeida Monfardini LP, Cunha AK, Rady P, et al. Prospective observational study of candidemia in São Paulo, Brazil: incidence rate, epidemiology, and predictors of mortality. Infect Control Hosp Epidemiol 2007;28:570-6.

36. Brunner HI, Gladman DD, Ibañez D, Urowitz MD, Silverman ED Difference in disease features between childhood-onset and adult-onset systemic lupus erythematosus. Arthritis Rheum 2008;58:556-62. 
37. Weng CT, Lee NY, Liu MF, Weng MY, Wu AB, Chang TW, et al. A retrospective study of catastrophic invasive fungal infections in patients with systemic lupus erythematosus from southern Taiwan. Lupus 2010;19:1204-9.

38. Gonzalez-Crespo MR, Gomez-Reino JJ. Invasive aspergillosis in systemic lupus erythematosus. Semin Arthritis Rheum 1995; 24:304-14.

39. Yeh TC, Liu HC, Hou JY, Chen KH, Huang TH, Chang CY, et al. Severe infections in children with acute leukemia undergoing intensive chemotherapy can successfully be prevented by ciprofloxacin, voriconazole, or micafungin prophylaxis. Cancer 2014;120:1255-62.
40. Groll AH, Castagnola E, Cesaro S, Dalle JH, Engelhard D, Hope W, et al; Fourth European Conference on Infections in Leukaemia; Infectious Diseases Working Party of the European Group for Blood Marrow Transplantation (EBMT-IDWP); Infectious Diseases Group of the European Organisation for Research and Treatment of Cancer (EORTC-IDG); International Immunocompromised Host Society (ICHS); European Leukaemia Net (ELN). Fourth European Conference on Infections in Leukaemia (ECIL-4): guidelines for diagnosis, prevention, and treatment of invasive fungal diseases in paediatric patients with cancer or allogeneic haemopoietic stem-cell transplantation. Lancet Oncol 2014;15:e327-40. 